\title{
Socio-behavioral factors, oral hygiene level and periodontitis prevalence in a 35-44-year-old Greek adult population: A cross-sectional survey
}

\author{
Iliana Diamanti ${ }^{1}$, Argy Polychronopoulou ${ }^{1}$, William Papaioannou ${ }^{1}$, Charis Theodoridis ${ }^{2}$, Vasileios Margaritis ${ }^{3}$, \\ Eleni Mamai-Homata ${ }^{1}$, Sotirios Kalfas ${ }^{2}$
}

\author{
${ }^{1}$ Department of Preventive and Community Dentistry, School of Dentistry, National and Kapodistrian University of Athens, \\ Athens, Greece \\ ${ }^{2}$ Department of Preventive Dentistry, Periodontology and Implant Biology, School of Dentistry, Aristotle University of Thessalo- \\ niki, Thessaloniki, Greece \\ ${ }^{3}$ School of Health Sciences, Walden University, Minneapolis, Minnesota, USA
}

Correspondence:

School of Dentistry

National and Kapodistrian University of Athens

2 Thivon Str, Goudi 115 27, Athens, Greece

idiamanti@dent.uoa.gr

Received: $18 / 04 / 202$

Accepted: $24 / 08 / 2021$

Diamanti I, Polychronopoulou A, Papaioannou W, Theodoridis C, Margaritis V, Mamai-Homata E, Kalfas S. Socio-behavioral factors, oral hygiene level and periodontitis prevalence in a 35-44-year-old Greek adult population: A cross-sectional survey. J Clin Exp Dent. 2021;13(10):e1021-9.

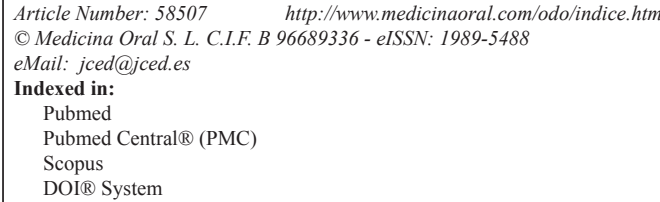

\begin{abstract}
Background: Sub-optimal oral hygiene is considered as a primary risk factor for periodontitis occurrence. Various socio-behavioral determinants may contribute either independently or by influencing adversely the oral hygiene $(\mathrm{OH})$ level. The aim of the present study was to examine the periodontal status of 35-44-year-old Greek adults and determine the contribution of risk indicators, including the socio-behavioral aspects and the population's oral hygiene level, on disease prevalence.

Material and Methods: In 1218 participants, Community Periodontal Index (CPI), Loss of Attachment (LoA) and simplified Oral Hygiene Index (OHI-S) were calculated. Multivariable regression models examined the effect of socio-behavioral factors with/without the inclusion of OHI-S level on pocket depth (PD) $\geq 4 \mathrm{~mm}$ and LoA $\geq 4 \mathrm{~mm}$ presence.

Results: $11.8 \%$ of the participants had healthy periodontium, whereas $37.4 \%$ and $5.7 \%$ presented with shallow and deep pocketing, respectively. $60.3 \%, 28.7 \%$, and $11.0 . \%$ of the adults demonstrated LoA $\leq 3 \mathrm{~mm}, 4-5 \mathrm{~mm}$, and $\geq 6 \mathrm{~mm}$, accordingly. Fair and poor oral hygiene significantly increased the likelihood for $\mathrm{PD} \geq 4 \mathrm{~mm}(\mathrm{OR}=4.8-20.3)$ and $\mathrm{LoA} \geq 4 \mathrm{~mm}(\mathrm{OR}=3.3-6.0)$ presence. 'Emergency-oriented dental visiting pattern' significantly elevated the chance for $\mathrm{PD} \geq 4 \mathrm{~mm}$ presence ( $\mathrm{OR}=1.7)$. 'Lower education level' 'urban location', and 'using an interdental brush' were significantly independently linked to $\mathrm{LoA} \geq 4 \mathrm{~mm}$ occurrence $(\mathrm{OR}=1.7-2.1,1.5$, and 2.0, respectively). Lower educated individuals demonstrated inferior oral hygiene status, which in turn elevated significantly the chances of $\mathrm{PD} \geq 4 \mathrm{~mm}$ presence. Smoking more than 10 cigarettes/day, emergency-oriented dental attendance pattern and not flossing were linked to worse oral hygiene levels, which consequently increased significantly the likelihood of LoA $\geq 4 \mathrm{~mm}$ occurrence.

Conclusions: Fair and poor oral hygiene contribute strongly to periodontitis occurrence. Various socio-behavioral factors may influence adversely oral hygiene maintenance, leading to periodontitis manifestations.
\end{abstract}

Key words: Community periodontal index, periodontal attachment loss, oral hygiene, adults 35-44, cross-sectional survey, socio-behavioral indicators. 


\section{Introduction}

Periodontal disease is recognized as a major oral pathological condition, reported as being a common entity among adult populations worldwide (1), although its quantification in a reproducible and comparable manner has been an ongoing challenge (2). It is reported to impact adversely the oral health-related quality of life of patients (3), and may lead to tooth loss with associated multidimensional impairment (4). Furthermore, periodontitis is well documented as a major contributor to systemic inflammation burden of the individual, which is strongly implicated in coronary artery disease, stroke and Type II diabetes (5). Also, the evidence regarding possible direct distal effects of periodontal bacteremia on adverse pregnancy outcomes, implies a significant association (6). Therefore, the estimation and understanding of periodontal disease occurrence at the population-level is regarded as highly important for all healthcare providers (7).

Similar to general health, profound disparities have been reported among populations concerning periodontal health (7). This variance may result from differences in examination protocols and periodontitis case definitions among studies, however, differences in demographic characteristics and levels of exposure to various risk factors among populations are thought to play a major role (1). Therefore, when evaluating periodontal disease manifestation in population field studies, risk analysis has to be performed (8). Related factors include demographic indicators such as gender (9), socio-economic position parameters, particularly education and income $(10,11)$, oral hygiene behaviors such as toothbrushing frequency (12) and the use of interdental cleaning devices (13), dental attendance patterns (14) and lifestyle dimensions such as smoking habits (15). Furthermore, clinical indicators, such as the oral hygiene level, have been strongly linked to periodontitis occurrence (16). Specifically, current evidence suggests a dose-response relationship between oral hygiene and periodontitis, whereas, the effect of oral hygiene has been estimated to be stronger than of other established risk factors, such as smoking (16). It may be hypothesized that the socio-behavioral risk factors influence periodontitis occurrence either independently, or through their adverse effect on the oral hygiene status, which in turn raises the probability for the disease establishment.

The present cross-sectional study aimed to examine the periodontal condition of 35-44-year-old Greek adults and assess the relative contribution of socio-demographic characteristics, dental attendance patterns, oral hygiene practices, and smoking habits in the presence of periodontitis, with or without taking into account the oral hygiene level of the adult population.

\section{Material and Methods}

A sample of 1218 35-44-year-old Greek adults was selected, in 2014 individuals being drawn from readily accessible primarily working population groups according to WHO pathfinder survey methodology for the specific age cluster, which ensures the participation of a satisfactory size of people that may present different disease prevalence (17). In order our results to be comparable with previous pathfinder surveys, sample collection has been held in a similar manner and in the same areas as then (18-21). Specifically, the survey covered three urban communities of different socio-economic backgrounds from the two larger metropolitan areas of Greece (Athens and Thessaloniki) and one urban and one rural community from each of five mainland counties, as well as four islands. (in total, 24 sites). Approximately 50 subjects were selected and examined at each site, as this is the standard size for each sampling site recommended as appropriate by the pathfinder sampling methodology established by WHO, in populations with high disease levels (17). In each sampling site, sampling points representing places where 35-44-year-old adults tend to gather were selected randomly (mainly working places in the public and in the private sector, but also places such as churches, culture clubs, public squares, as well as private properties), according to the layout of the region under examination, rural or urban. The average drop-out rate was $20 \%$. It was estimated that the minimum size of the sample to report outcomes of interest, with $95 \%$ certainty and an acceptable error of $3 \%$, was 897 subjects. A $30 \%$ prevalence of periodontitis was used in this calculation (22), as it referred to the observed in a previous pathfinder survey percentage of 35-44-year-old adults demonstrating at least one tooth site with periodontal pocket depth $\geq 4 \mathrm{~mm}$ (18).

Calibration of the eight participating examiners took place against a reference examiner that was considered as the 'gold standard'. Inter-examiner agreement as described by weighted \% agreement was estimated to be $85 \%-96 \%$ and $83 \%-90 \%$ for the loss of attachment (LoA) and the Community Periodontal Index (CPI) outcomes, respectively. All examinations were carried out under artificial light using dental mirrors and the WHO CPI periodontal probe. Moisture control was managed by using cotton rolls and sterilized gauze, if needed.

Clinical examination collected data describing gingival bleeding on probing, presence of calculus, periodontal pocketing (pocket depth, PD) and associated loss of periodontal attachment (recorded only if: cemento-enamel junction non-visible and $\mathrm{CPI}=4$, or cemento-enamel junction visible) on teeth $11,31,16 / 17,26 / 27,36 / 37$ and $46 / 47$ according to the WHO recommendations (17). Based on the clinical assessment, Community Periodontal Index (CPI) and loss of periodontal attachment (LoA) 
categorized in multiples of $\mathrm{mm}$ (i.e. $0-3 \mathrm{~mm}, 4-5 \mathrm{~mm}$, $6-8 \mathrm{~mm}, 9-11 \mathrm{~mm}$ and $12+\mathrm{mm}$ ) were calculated. Furthermore, for the calculation of the simplified oral hygiene index $(\mathrm{OHI}-\mathrm{S})$ the proportion of the buccal surfaces of teeth 11,31, 16 and 26, as well as the lingual surfaces of teeth 36 and 46 covered with soft debris or calculus deposits was recorded. Subsequently, OHI-S was categorized into good (OHI-S=0.0-1.2), fair (OHI-S=1.3-3.0) or poor $(\mathrm{OHI}-\mathrm{S}=3.1-6.0)(22,23)$.

Socio-demographic and behavioral data were collected through structured questionnaires. Demographic characteristics (gender, urban/rural location) and socio-economic status parameters (level of education categorized into: up to lower secondary, upper secondary/non-university tertiary, university and income divided into: $(\leq 590 €, 591-1200 €$ and $>1200 €)$ were recorded. Periodontal health-related behaviors were described by dental attendance pattern (dental visit when in pain or other emergencies/for treatment/for a check-up and preventive procedures), the time elapsed since last dental visit (up to one year/more than a year), toothbrushing frequency (less than twice per day/twice per day), use of dental floss (no/yes), use of interdental brush (no/yes) and tobacco use (no/1-10 cigarettes per day $>10$ cigarettes per day).

Regarding missing data, no correction for non-participation or non-response was applied. Descriptive analysis of CPI, and LoA categories by each socio-demographic/behavioral variable was performed with the use of non-parametric tests (Mann-Whitney and Kruskal-Wallis tests). Univariable and multivariable logistic regression models were used to identify the socio-demographic, behavioral, and clinical indicators for the presence of at least one tooth site with pocket depth $\geq 4 \mathrm{~mm}$ and with loss of attachment $\geq 4 \mathrm{~mm}$. The analysis was performed either by adjusting for the oral hygiene status (OHI-S) of the participants or not, to investigate if the inclusion of a primary clinical indicator attenuates or not the association of the socio-behavioral variables with the periodontal disease outcome. Also, a similar logistic regression analysis was carried out to examine the association between the oral hygiene status of the participants and the socio-behavioral co-variates. In the multivariable analyses, only the univariably significant parameters $(\mathrm{p} \leq 0.05)$ were included. Results have been presented with odds ratios (OR) and $95 \%$ confidence intervals (CI) with the level of significance set at $p \leq 0.05$. In all regression analyses the comparisons were estimated against one of the categories that was considered as reference. Data were processed and analyzed with IBM SPSS Statistics (PC version 26.0).

\section{Results}

Descriptive analysis of CPI, and LoA categories is presented in Tables 1, and 2, respectively. The percentage of individuals with healthy periodontal condition was
$11.8 \%$, whereas categorization by worst CPI scores revealed $6.4 \%, 38.6 \%, 37.4 \%$, and $5.7 \%$ of those, presenting with bleeding, calculus, shallow, and deep periodontal pockets, accordingly. Also, the mean number of sextants with a healthy periodontium was 2.0 , whereas the corresponding values for shallow and deep periodontal pockets were 1.2 and 0.1 , respectively. Furthermore, a mean of 0.1 sextants were excluded from the analysis, as they contained less than two teeth. The analysis of CPI categories by socio-demographic, behavioral and clinical indicators revealed a significantly healthier periodontium among women, urban residents, individuals with higher educational attainment, higher monthly income, frequent dental attenders, non-emergency-oriented dental visitors, dental floss users, better oral hygiene status achievers, non-smokers or non-heavy smokers $(\leq 10$ cigarettes per day), and among those who brushed their teeth twice per day.

The $\%$ percentage of subjects with $\mathrm{LoA} \leq 3 \mathrm{~mm}$ was 60.3 , whereas respective \% percentages for $\mathrm{LoA}=4-5 \mathrm{~mm}$, $6-8 \mathrm{~mm}, 9-11 \mathrm{~mm}$ and $\geq 12 \mathrm{~mm}$ were $28.7,9.4,1.1$, and 0.5 . Also, the majority of sextants presented loss of attachment $\leq 3 \mathrm{~mm}$ (mean: 4.8 ), whereas a mean of 0.9 and 0.2 sextants demonstrated loss of attachment $4-5$ and $\geq 6 \mathrm{~mm}$, accordingly. The analysis of LoA categories by socio-demographic and behavioral indicators, showed that attachment loss was significantly less common and less severe among higher educated individuals, frequent dental attenders, non-emergency - oriented dental visitors, as well as among participants who used dental floss, did not use an interdental brush, maintained better oral hygiene levels and refrained from tobacco use or smoked up to 10 cigarettes per day.

Univariate analysis of the $\mathrm{PD} \geq 4 \mathrm{~mm}$ distribution (Table 3 ) showed that all independent variables were significantly related to the outcome, except for the 'last dental visit', the 'toothbrushing frequency' and the 'use of interdental brush' parameters. The unadjusted for the OHI-S multivariable regression model referring to $\mathrm{PD} \geq 4 \mathrm{~mm}$ as the outcome (Table 3 ) revealed that, a significantly increased probability of presenting at least one tooth with $\mathrm{PD} \geq 4 \mathrm{~mm}$ was linked to lower education attainment and visiting the dentist mainly for emergency treatment. However, in the adjusted for the OHI-S model (Table 3), only emergency-oriented dental visits continued to be significantly independently associated with the presence of $\mathrm{PD} \geq 4 \mathrm{~mm}$. In the latter model, possessing fair or poor oral hygiene status increased the probability by nearly 4 and 20 times accordingly, of being detected with $\mathrm{PD} \geq 4 \mathrm{~mm}$.

In the univariable analysis of the $\mathrm{LoA} \geq 4 \mathrm{~mm}$ distribution (Table 4), only the 'gender', the 'monthly income' and the 'toothbrushing frequency' parameters were not associated with the outcome. The unadjusted for the OHI-S multivariable regression model referring to $\mathrm{LoA} \geq 4 \mathrm{~mm}$ 
Table 1: Periodontal conditions of 35-44-year-olds Greek adults measured by CPI, according to socio-demographic, behavioral, and clinical parameters.

\begin{tabular}{|c|c|c|c|c|c|c|c|}
\hline & \multicolumn{6}{|c|}{$\%$ of persons who have as highest score } & \multirow[t]{2}{*}{$p$-value } \\
\hline & $\mathbf{N}^{\dagger}$ & $\begin{array}{c}\text { 0- } \\
\text { Healthy }\end{array}$ & $\begin{array}{c}\text { 1- } \\
\text { Bleeding }\end{array}$ & $\begin{array}{c}2- \\
\text { Calculus }\end{array}$ & $\begin{array}{c}3- \\
\mathrm{PD}=4-5 \mathrm{~mm}\end{array}$ & $\begin{array}{c}4- \\
P D \geq 6 \mathrm{~mm}\end{array}$ & \\
\hline \multicolumn{8}{|l|}{ Area of residence } \\
\hline Rural & 411 & 10.2 & 4.4 & 36.3 & 41.1 & 8.0 & \multirow[t]{2}{*}{$<0.001^{*}$} \\
\hline Urban & 807 & 12.6 & 7.4 & 39.8 & 35.6 & 4.6 & \\
\hline \multicolumn{8}{|l|}{ Gender } \\
\hline Male & 551 & 10.3 & 5.8 & 36.8 & 39.6 & 7.4 & \multirow[t]{2}{*}{$<0.01^{*}$} \\
\hline Female & 667 & 13.0 & 6.9 & 40.0 & 35.7 & 4.3 & \\
\hline \multicolumn{8}{|l|}{ Level of education } \\
\hline Up to lower secondary & 158 & 5.1 & 1.3 & 31.0 & 50.6 & 12.0 & \multirow[t]{3}{*}{$<0.001^{* *}$} \\
\hline Upper secondary/non-university tertiary & 683 & 9.7 & 6.6 & 38.9 & 38.7 & 6.1 & \\
\hline University & 368 & 19.0 & 8.2 & 40.8 & 29.6 & 2.4 & \\
\hline \multicolumn{8}{|l|}{ Monthly income } \\
\hline$\leq 590 €$ & 236 & 8.1 & 5.1 & 38.6 & 41.5 & 6.8 & \multirow[t]{3}{*}{$<0.01^{* *}$} \\
\hline $591 €-1200 €$ & 714 & 12.0 & 7.0 & 36.8 & 38.1 & 6.0 & \\
\hline$>1200 €$ & 230 & 16.1 & 6.5 & 41.7 & 31.7 & 3.9 & \\
\hline \multicolumn{8}{|l|}{ Reason for dental visit } \\
\hline Pain & 408 & 6.8 & 4.1 & 32.0 & 47.8 & 9.2 & \multirow[t]{3}{*}{$<0.001^{* *}$} \\
\hline Treatment & 252 & 10.3 & 4.0 & 45.2 & 36.5 & 4.0 & \\
\hline Check-up/Prevention & 532 & 15.8 & 9.0 & 40.6 & 30.8 & 3.8 & \\
\hline \multicolumn{8}{|l|}{ Last dental visit } \\
\hline$\leq 1$ year & 845 & 13.4 & 7.0 & 38.3 & 36.6 & 4.7 & \multirow[t]{2}{*}{$<0.01^{*}$} \\
\hline$>1$ year & 316 & 8.2 & 4.1 & 40.5 & 38.9 & 8.2 & \\
\hline \multicolumn{8}{|l|}{ Toothbrushing frequency } \\
\hline$<$ twice per day & 649 & 8.0 & 4.8 & 42.2 & 38.2 & 6.8 & \multirow[t]{2}{*}{$<0.001^{*}$} \\
\hline Twice per day & 563 & 16.3 & 8.0 & 34.5 & 36.8 & 4.4 & \\
\hline \multicolumn{8}{|l|}{ Use of dental floss } \\
\hline No & 820 & 9.3 & 4.8 & 40.0 & 39.0 & 7.0 & \multirow[t]{2}{*}{$<0.001^{*}$} \\
\hline Yes & 382 & 17.5 & 9.7 & 35.9 & 34.6 & 2.4 & \\
\hline \multicolumn{8}{|l|}{ Use of interdental brush } \\
\hline No & 1035 & 11.7 & 5.7 & 39.7 & 36.9 & 6.0 & \multirow[t]{2}{*}{$N S^{*}$} \\
\hline Yes & 165 & 13.3 & 10.3 & 29.7 & 42.4 & 4.2 & \\
\hline \multicolumn{8}{|l|}{ Smoking } \\
\hline No & 686 & 14.0 & 7.9 & 38.0 & 35.4 & 4.7 & \multirow[t]{3}{*}{$<0.001^{* *}$} \\
\hline $1-10$ cigarettes/day & 196 & 14.3 & 6.6 & 36.7 & 39.3 & 3.1 & \\
\hline$>10$ cigarettes/day & 324 & 5.9 & 3.1 & 41.0 & 40.4 & 9.6 & \\
\hline \multicolumn{8}{|l|}{ OHI-S } \\
\hline Good (0.0-1.2) & 632 & 22.5 & 10.0 & 45.3 & 20.4 & 1.9 & \multirow[t]{3}{*}{$<0.001^{* *}$} \\
\hline Fair (1.3-3.0) & 463 & 0.2 & 3.2 & 36.1 & 53.3 & 7.1 & \\
\hline Poor (3.1-6.0) & 119 & 0.0 & 0.0 & 14.3 & 66.4 & 19.3 & \\
\hline Total & 1218 & 11.8 & 6.4 & 38.6 & 37.4 & 5.7 & \\
\hline
\end{tabular}

$\uparrow$ Number of observations may be less than total for some variables due to missing data. *Mann-Whitney U test. **Kruskal-Wallis test. NS: Not Significant. 
Table 2: Periodontal destruction of 35-44-year-olds Greek adults measured by Loss of Attachment (LoA), according to socio-demographic, behavioral, and clinical parameters.

\begin{tabular}{|c|c|c|c|c|c|c|c|}
\hline & \multicolumn{6}{|c|}{$\%$ of persons who have as highest score } & \multirow[t]{2}{*}{$p$-value } \\
\hline & $\mathbf{N}^{\dagger}$ & $\begin{array}{c}0- \\
0-3 \mathrm{~mm}\end{array}$ & $\begin{array}{c}1- \\
4-5 \mathrm{~mm}\end{array}$ & $\begin{array}{c}2- \\
6-8 \mathrm{~mm}\end{array}$ & $\begin{array}{c}3- \\
9-11 \mathrm{~mm}\end{array}$ & $\begin{array}{c}4- \\
\geq 12 \mathrm{~mm}\end{array}$ & \\
\hline \multicolumn{8}{|l|}{ Area of residence } \\
\hline Rural & 411 & 64.2 & 22.6 & 11.4 & 1.2 & 0.5 & \multirow[t]{2}{*}{$N S^{*}$} \\
\hline Urban & 807 & 58.4 & 31.8 & 8.3 & 1.0 & 0.5 & \\
\hline \multicolumn{8}{|l|}{ Gender } \\
\hline Male & 551 & 58.8 & 27.4 & 11.1 & 2.0 & 0.7 & \multirow[t]{2}{*}{$N S^{*}$} \\
\hline Female & 667 & 61.6 & 29.8 & 7.9 & 0.3 & 0.3 & \\
\hline \multicolumn{8}{|l|}{ Level of education } \\
\hline Up to lower secondary & 158 & 44.3 & 33.5 & 18.4 & 1.9 & 1.9 & \multirow[t]{3}{*}{$<0.001^{* *}$} \\
\hline Upper secondary/non-university tertiary & 683 & 58.1 & 30.3 & 10.1 & 1.0 & 0.4 & \\
\hline University & 368 & 71.7 & 23.1 & 4.3 & 0.8 & 0.0 & \\
\hline \multicolumn{8}{|l|}{ Monthly income } \\
\hline$\leq 590 €$ & 236 & 55.1 & 31.8 & 12.3 & 0.0 & 0.8 & \multirow[t]{3}{*}{$N S^{* *}$} \\
\hline $591 €-1200 €$ & 714 & 61.6 & 27.5 & 8.7 & 1.7 & 0.6 & \\
\hline$>1200 €$ & 230 & 60.9 & 30.4 & 8.7 & 0.0 & 0.0 & \\
\hline \multicolumn{8}{|l|}{ Reason for dental visit } \\
\hline Pain & 412 & 52.2 & 30.8 & 14.6 & 1.2 & 1.2 & \multirow[t]{3}{*}{$<0.001^{* *}$} \\
\hline Treatment & 252 & 58.7 & 34.5 & 6.0 & 0.8 & 0.0 & \\
\hline Check-up/Prevention & 532 & 66.7 & 25.0 & 7.1 & 0.9 & 0.2 & \\
\hline \multicolumn{8}{|l|}{ Last dental visit } \\
\hline$\leq 1$ year & 845 & 63.1 & 27.7 & 7.8 & 1.1 & 0.4 & \multirow[t]{2}{*}{$<0.01^{*}$} \\
\hline$>1$ year & 316 & 54.7 & 30.1 & 13.3 & 0.9 & 0.9 & \\
\hline \multicolumn{8}{|l|}{ Toothbrushing frequency } \\
\hline$<$ twice per day & 647 & 58.7 & 29.4 & 10.2 & 1.2 & 0.5 & \multirow[t]{2}{*}{$N S^{*}$} \\
\hline Twice per day & 563 & 62.3 & 28.1 & 8.3 & 0.7 & 0.5 & \\
\hline \multicolumn{8}{|l|}{ Use of dental floss } \\
\hline No & 820 & 57.4 & 29.9 & 10.7 & 1.2 & 0.7 & \multirow[t]{2}{*}{$<0.001^{*}$} \\
\hline Yes & 382 & 67.3 & 26.7 & 5.5 & 0.5 & 0.0 & \\
\hline \multicolumn{8}{|l|}{ Use of interdental brush } \\
\hline No & 1035 & 61.9 & 27.2 & 9.4 & 0.9 & 0.6 & \multirow[t]{2}{*}{$<0.05^{*}$} \\
\hline Yes & 165 & 51.5 & 37.0 & 9.7 & 1.8 & 0.0 & \\
\hline \multicolumn{8}{|l|}{ Smoking } \\
\hline No & 686 & 63.0 & 27.4 & 8.3 & 1.2 & 0.1 & \multirow[t]{3}{*}{$0.001^{* *}$} \\
\hline 1-10 cigarettes/day & 196 & 63.8 & 28.6 & 6.1 & 0.5 & 1.0 & \\
\hline$>10$ cigarettes/day & 324 & 52.8 & 31.5 & 13.9 & 0.9 & 0.9 & \\
\hline \multicolumn{8}{|l|}{ OHI-S } \\
\hline Good (0.0-1.2) & 632 & 75.8 & 19.6 & 4.1 & 0.3 & 0.0 & \multirow[t]{3}{*}{$<0.001^{* *}$} \\
\hline Fair (1.3-3.0) & 463 & 46.4 & 39.3 & 12.3 & 1.5 & 0.4 & \\
\hline Poor (3.1-6.0) & 119 & 32.8 & 37.0 & 24.4 & 3.4 & 2.5 & \\
\hline Total & 1218 & 60.3 & 28.7 & 9.4 & 1.1 & 0.5 & \\
\hline
\end{tabular}

$\dagger$ Number of observations may be less than total for some variables due to missing data. *Mann-Whitney U test. **Kruskal-Wallis test. NS: Not Significant. 
Table 3: Effect of socio-demographic and behavioral factors on the probability of the presence of at least one tooth with periodontal pocket depth (PD) $\geq 4 \mathrm{~mm}$ of 35-44-year-old Greek adults a) in univariable logistic regression models and b) in multivariable logistic regression models, either unadjusted $(\mathrm{n}=1136)$ or adjusted $(\mathrm{n}=1132)$ for the oral hygiene $(\mathrm{OH})$ status of the participants.

\begin{tabular}{|c|c|c|c|c|c|c|c|c|c|}
\hline & \multicolumn{3}{|c|}{$\begin{array}{c}\mathrm{PD} \geq 4 \mathrm{~mm} \text { (univariable } \\
\text { models) }\end{array}$} & \multicolumn{3}{|c|}{$\begin{array}{c}\text { Unadjusted for } \mathrm{OH} \text { status } \\
\text { multivariable } \mathrm{PD} \geq 4 \mathrm{~mm} \\
\text { model }\end{array}$} & \multicolumn{3}{|c|}{$\begin{array}{l}\text { Adjusted for } O H \text { status } \\
\text { multivariable } P D \geq 4 \mathrm{~mm} \\
\text { model }\end{array}$} \\
\hline & OR & $95 \% \mathrm{CI}$ & $p$-value & OR & $95 \% \mathrm{CI}$ & $p$-value & OR & $95 \% \mathrm{CI}$ & $p$-value \\
\hline \multicolumn{10}{|l|}{ Area of residence } \\
\hline Rural & 1 & & & 1 & & & 1 & & \\
\hline Urban & 0.69 & $0.55-0.88$ & 0.003 & 0.87 & $0.67-1.13$ & NS & 0.82 & $0.61-1.09$ & NS \\
\hline \multicolumn{10}{|l|}{ Gender } \\
\hline Female & 1 & & & 1 & & & 1 & & \\
\hline Male & 1.33 & $1.06-1.67$ & 0.015 & 1.26 & $0.98-1.63$ & NS & 1.05 & $0.79-1.39$ & NS \\
\hline \multicolumn{10}{|l|}{ Level of education } \\
\hline University & 1 & & & 1 & & & 1 & & \\
\hline $\begin{array}{l}\text { Upper secondary/non-univ } \\
\text { tertiary }\end{array}$ & 1.72 & $1.32-2.24$ & $<0.001$ & 1.44 & $1.07-1.93$ & 0.016 & 1.24 & $0.90-1.71$ & NS \\
\hline Up to lower secondary & 3.56 & $2.41-5.25$ & $<0.001$ & 2.53 & $1.60-3.98$ & $<0.001$ & 1.49 & $0.89-2.48$ & NS \\
\hline \multicolumn{10}{|l|}{ Monthly income } \\
\hline$\leq 590 €$ & 1 & & & 1 & & & 1 & & \\
\hline $591 €-1200 €$ & 0.85 & $0.63-1.14$ & NS & 1.09 & $0.79-1.52$ & NS & 1.21 & $0.84-1.74$ & NS \\
\hline$>1200 €$ & 0.59 & $0.41-0.86$ & 0.006 & 0.95 & $0.62-1.46$ & NS & 1.32 & $0.82-2.12$ & NS \\
\hline \multicolumn{10}{|l|}{ Reason for dental visit } \\
\hline Check-up/Prevention & 1 & & & 1 & & & 1 & & \\
\hline Treatment & 1.29 & $0.95-1.75$ & NS & 1.08 & $0.77-1.50$ & NS & 0.90 & $0.63-1.30$ & NS \\
\hline Pain/Acute problem & 2.51 & $1.93-3.27$ & $<0.001$ & 1.98 & $1.48-2.64$ & $<0.001$ & 1.74 & $1.26-2.39$ & $<0.001$ \\
\hline \multicolumn{10}{|l|}{ Last dental visit } \\
\hline$\leq 1$ year & 1 & & & & & & & & \\
\hline$>1$ year & 1.27 & $0.98-1.64$ & NS & & & & & & \\
\hline \multicolumn{10}{|l|}{ Toothbrushing frequency } \\
\hline$<$ twice per day & 1 & & & & & & & & \\
\hline Twice per day & 0.86 & $0.68-1.08$ & NS & & & & & & \\
\hline \multicolumn{10}{|l|}{ Use of dental floss } \\
\hline No & 1 & & & 1 & & & 1 & & \\
\hline Yes & 0.69 & $0.54-0.88$ & 0.003 & 0.91 & $0.69-1.20$ & NS & 1.08 & $0.80-1.46$ & NS \\
\hline \multicolumn{10}{|l|}{ Use of interdental brush } \\
\hline No & 1 & & & & & & & & \\
\hline Yes & 1.17 & $0.84-1.62$ & NS & & & & & & \\
\hline \multicolumn{10}{|l|}{ Smoking } \\
\hline No & 1 & & & 1 & & & 1 & & \\
\hline 1-10 cigarettes/day & 1.10 & $0.80-1.52$ & NS & 1.21 & $0.86-1.70$ & NS & 1.12 & $0.77-1.62$ & NS \\
\hline$>10$ cigarettes/day & 1.50 & $1.15-1.95$ & 0.003 & 1.21 & $0.91-1.61$ & NS & 0.99 & $0.72-1.36$ & NS \\
\hline \multicolumn{10}{|l|}{ OHI-S } \\
\hline Good (0.0-1.2) & 1 & & & & & & 1 & & \\
\hline Fair (1.3-3.0) & 5.33 & $4.09-6.94$ & $<0.001$ & & & & 4.76 & $3.59-6.32$ & $<0.001$ \\
\hline Poor (3.1-6.0) & 20.89 & $12.10-36.09$ & $<0.001$ & & & & 20.32 & $10.79-38.29$ & $<0.001$ \\
\hline
\end{tabular}

NS: Not Significant 
Table 4: Effect of socio-demographic and behavioral factors on the probability of the presence of at least one tooth with attachment loss (LoA) $\geq 4 \mathrm{~mm}$ of 35-44-year-old Greek adults a) in univariable logistic regression models and b) in multivariable logistic regression models, either unadjusted $(\mathrm{n}=1116)$ or adjusted $(\mathrm{n}=1113)$ for the oral hygiene $(\mathrm{OH})$ status of the participants.

\begin{tabular}{|c|c|c|c|c|c|c|c|c|c|}
\hline & \multicolumn{3}{|c|}{$\begin{array}{c}\text { LoA } \geq 4 \text { mm (univariable } \\
\text { models) }\end{array}$} & \multicolumn{3}{|c|}{$\begin{array}{l}\text { Unadjusted for } \mathrm{OH} \text { status } \\
\text { multivariable } \mathrm{LoA} \geq 4 \mathrm{~mm} \\
\text { model }\end{array}$} & \multicolumn{3}{|c|}{$\begin{array}{l}\text { Adjusted for } \mathrm{OH} \text { status } \\
\text { multivariable } \mathrm{LoA} \geq 4 \mathrm{~mm} \\
\text { model }\end{array}$} \\
\hline & OR & $95 \% \mathrm{CI}$ & $p$-value & OR & $95 \% \mathrm{CI}$ & $p$-value & OR & $95 \% \mathrm{CI}$ & $p$-value \\
\hline \multicolumn{10}{|l|}{ Area of residence } \\
\hline Rural & 1 & & & 1 & & & 1 & & \\
\hline Urban & 1.28 & $1.00-1.64$ & 0.048 & 1.54 & $1.17-2.03$ & 0.002 & 1.51 & $1.13-2.01$ & 0.005 \\
\hline \multicolumn{10}{|l|}{ Gender } \\
\hline Female & 1 & & & & & & & & \\
\hline Male & 1.13 & $0.89-1.42$ & NS & & & & & & \\
\hline \multicolumn{10}{|l|}{ Level of education } \\
\hline University & 1 & & & 1 & & & 1 & & \\
\hline $\begin{array}{l}\text { Upper secondary/non- } \\
\text { univ tertiary }\end{array}$ & 1.83 & $1.39-2.40$ & $<0.001$ & 1.89 & $1.40-2.54$ & $<0.001$ & 1.65 & $1.21-2.26$ & 0.002 \\
\hline Up to lower secondary & 3.19 & $2.17-4.70$ & $<0.001$ & 3.09 & $1.99-4.79$ & $<0.001$ & 2.11 & $1.32-3.38$ & 0.002 \\
\hline \multicolumn{10}{|l|}{ Monthly income } \\
\hline$\leq 590 €$ & 1 & & & & & & & & \\
\hline $591 €-1200 €$ & 0.76 & $0.57-1.03$ & NS & & & & & & \\
\hline$>1200 €$ & 0.79 & $0.55-1.14$ & NS & & & & & & \\
\hline \multicolumn{10}{|l|}{ Reason for dental visit } \\
\hline Check-up/Prevention & 1 & & & 1 & & & 1 & & \\
\hline Treatment & 1.41 & $1.04-1.92$ & 0.029 & 1.24 & $0.88-1.75$ & NS & 1.16 & $0.81-1.67$ & NS \\
\hline Pain/Acute problem & 1.84 & $1.41-2.39$ & $<0.001$ & 1.55 & $1.15-2.10$ & 0.004 & 1.34 & $0.97-1.84$ & NS \\
\hline \multicolumn{10}{|l|}{ Last dental visit } \\
\hline$\leq 1$ year & 1 & & & 1 & & & 1 & & \\
\hline$>1$ year & 1.41 & $1.09-1.84$ & 0.010 & 1.23 & $0.92-1.64$ & NS & 1.10 & $0.82-1.49$ & NS \\
\hline \multicolumn{10}{|l|}{ Toothbrushing frequency } \\
\hline$<$ twice per day & 1 & & & & & & & & \\
\hline Twice per day & 0.86 & $0.68-1.08$ & NS & & & & & & \\
\hline \multicolumn{10}{|l|}{ Use of dental floss } \\
\hline No & 1 & & & 1 & & & 1 & & \\
\hline Yes & 0.66 & $0.51-0.85$ & 0.001 & 0.70 & $0.53-0.94$ & 0.018 & 0.79 & $0.58-1.07$ & NS \\
\hline \multicolumn{10}{|l|}{ Use of interdental brush } \\
\hline No & 1 & & & 1 & & & 1 & & \\
\hline Yes & 1.53 & $1.10-2.13$ & 0.011 & 1.98 & $1.35-2.89$ & $<0.001$ & 2.00 & $1.35-2.98$ & $<0.001$ \\
\hline \multicolumn{10}{|l|}{ Smoking } \\
\hline No & 1 & & & 1 & & & 1 & & \\
\hline 1-10 cigarettes/day & 0.97 & $0.70-1.34$ & NS & 0.97 & $0.68-1.39$ & NS & 0.94 & $0.64-1.36$ & NS \\
\hline$>10$ cigarettes/day & 1.52 & 1.17-1.99 & 0.002 & 1.44 & $1.08-1.93$ & 0.014 & 1.25 & $0.92-1.70$ & NS \\
\hline \multicolumn{10}{|l|}{ OHI-S } \\
\hline Good (0.0-1.2) & 1 & & & & & & 1 & & \\
\hline Fair (1.3-3.0) & 3.64 & $2.81-4.72$ & $<0.001$ & & & & 3.30 & $2.50-4.37$ & $<0.001$ \\
\hline Poor (3.1-6.0) & 6.48 & $4.24-9.90$ & $<0.001$ & & & & 6.00 & $3.63-9.92$ & $<0.001$ \\
\hline
\end{tabular}

NS: Not Significant 
as the outcome (Table 4) revealed that LoA $\geq 4 \mathrm{~mm}$ probability was significantly independently associated with urban location, lower education attainment, emergency-oriented dental visits, not using dental floss, using an interdental brush and smoking $>10$ cigarettes per day. In the adjusted for the OHI-S regression analysis (Table 4), only the location, the education level and the use of interdental brush indicators demonstrated a significant independent association. In the latter model, achieving fair or poor oral hygiene status increased the probability by 2.3 and 5.0 times respectively of being detected with LoA $\geq 4 \mathrm{~mm}$.

Considering the oral hygiene status of the participants, about half of the 35-44-year-olds (52.1\%) had good oral hygiene, $38.1 \%$ fair, and $9.8 \%$ poor. Male gender ( $p=0.001)$, education up to lower secondary $(p<0.001)$ or upper secondary/non university tertiary level $(p=0.011)$, monthly income $<590$ euros $(p<0.001)$ or between 590 and 1200 euros $(p=0.008)$, infrequent $(>1$ year $)$ $(p=0.013)$ or emergency treatment-oriented $(p=0.016)$ dental attendance pattern, toothbrushing $<2$ times per day $(p=0.012)$, not flossing $(p=0.009)$ and heavy smoking ( $>10$ cigarettes per day) $(p=0.001)$ were significantly independently linked to worse levels of oral hygiene.

\section{Discussion}

The prevalence of periodontal disease observed in Greek 35-44-year-old adults is in line with analogous evidence from other European countries. In Western Europe, among 35-44-year-old adults, the pr evalence of periodontal disease was estimated for $\mathrm{PD} \geq 4 \mathrm{~mm}$ between $12.6 \%$ in Sweden (2003) and 73.2\% in Germany (2005) whereas for $\mathrm{LoA} \geq 4 \mathrm{~mm}$ between $20.0 \%$ in Denmark (2001) and $83.9 \%$ in Germany (2005) (8). Regarding the trends in the disease occurrence, a declining pattern could be demonstrated in the UK as the proportion of 35-44-year-old adults who manifested $\mathrm{PD} \geq 4 \mathrm{~mm}$ decreased from 59\% to 43\% between 1998 and 2009 (8) and in Germany, as the average number of teeth per adult with $\mathrm{PD} \geq 4 \mathrm{~mm}$ diminished from 6.3 to 4.8 between 2005-2014 (4). However, in Greece, the proportion of $35-44$-year-old adults presenting shallow or deep pocketing increased from $24.2 \%$ to $37.3 \%$ and from $3.3 \%$ to $5.6 \%$, respectively, between 2005 and 2014 (18).

In the present study, a strong association between oral hygiene levels and periodontitis was observed, as fair and poor oral hygiene increased the probability of $P D \geq 4 \mathrm{~mm}$ presence by 3.8 and 19.3 times and of $L o A \geq 4 \mathrm{~mm}$ presence by 2.3 and 5.0 times, respectively, compared with good oral hygiene. These observations accord with recent meta-analysis evidence, where fair and poor oral hygiene were found to significantly increase the risk of having periodontitis by two- and five-fold, accordingly, compared with good oral hygiene (16).

In the adjusted for the OHI-S level of the 35-44-year- old adults, models, different covariates remained independently significant. Specifically, 'emergency-oriented dental visits' were associated with $\mathrm{PD} \geq 4 \mathrm{~mm}$ outcome, whereas 'urban location', 'low education level' and 'using an interdental brush' were associated with LoA $\geq 4 \mathrm{~mm}$ outcome. Seeking dental services primarily when in pain has been associated with worse periodontal health, and it has been suggested that check-up dental visits on a regular basis help to maintain periodontal health and control disease progression (14). Rurality has been reported as a potential risk factor for periodontal disease $(8,11)$. However, as the multilevel nature of the area effect cannot place it as an all-suitable factor when it comes to health outcomes (10), it might be that urban-related environmental stress contributed adversely to periodontal health status. Low education level has been consistently associated with an increased probability for periodontitis (10), and the present study confirmed this observation. Although in prospective studies, interdental brushing was not associated with changes in periodontal pocketing (24), in the present survey Lo $\mathrm{A} \geq 4 \mathrm{~mm}$ was significantly higher among participants who used an interdental brush. This can be considered as an elaborate oral health behavior, presumably requiring high oral health literacy; therefore, it appears possible that the minority group (14\%) who adopted that habit, mostly included people that were already undergoing periodontally-related treatment, and thereupon were instructed to do so.

Several variables, specifically, 'lower education level' for the $\mathrm{PD} \geq 4 \mathrm{~mm}$ model, as well as 'urgent-oriented dental visits', 'not flossing' and 'smoking more than 10 cigarettes/day' for the LoA $\geq 4 \mathrm{~mm}$ model, were significantly independently associated with periodontitis presence only in the un-adjusted for the OHI-S regression analysis, whereas their association with the respective $\mathrm{PD} \geq 4 \mathrm{~mm}$ or $\mathrm{LoA} \geq 4 \mathrm{~mm}$ outcomes attenuated in the fully adjusted models. As adults with lower education attainment, those who paid dental visits primarily when in pain, did not floss, or smoked heavily demonstrated worse oral hygiene levels, it is reasonable to suggest that oral hygiene status had a mediating effect in the association between these parameters and each respective outcome.

The present study is based on the presentation and analysis of cross-sectional data therefore, causal relationships cannot be drawn from our results. Also, on account of the fact that the simplified sampling methodology developed by WHO has been followed (17), our sample, albeit large, cannot be characterized as random. Nevertheless, it may be regarded as illustrative of the whole population of the 35-44 age cluster, since it ensures the participation of a satisfactory size of people living in representative urban and rural areas of Greece and additionally, it offers the necessary comparability with previous 
studies conducted likewise. Furthermore, a pragmatic instead of a case definition of periodontitis was utilized, because of the nature of reporting the clinical assessment which was gathered for surveillance purposes.

In conclusion, oral hygiene level appears to play a key role in the periodontal status of 35-44-year-old adults, as fair and poor oral hygiene were the strongest indicators of periodontitis presence. Various socio-behavioural factors may contribute to inadequate oral hygiene maintenance, leading to periodontitis manifestations.

\section{References}

1. Holde GE, Oscarson N, Trovik TA, Tillberg A, Jönsson B. Periodontitis Prevalence and Severity in Adults: A Cross-Sectional Study in Norwegian Circumpolar Communities. J Periodontol. 2017;88:1012-22. 2. Kassebaum NJ, Bernabé E, Dahiya M, Bhandari B, Murray CJ, Marcenes W. Global burden of severe periodontitis in 1990-2010: a systematic review and meta-regression. J Dent Res. 2014;93:1045-53. 3. Theodoridis C, Violesti A, Nikiforidou M, Menexes GC, Vouros ID. Short-Term Impact of Non-Surgical and Surgical Periodontal Therapy on Oral Health-Related Quality of Life in a Greek Population-A Prospective Cohort Study. Dent J (Basel). 2020;8:54.

4. Schwendicke F, Krois J, Kocher T, Hoffmann T, Micheelis W, Jordan RA. More teeth in more elderly: Periodontal treatment need in Germany 1997-2030. J Clin Periodontol. 2018;45:1400-07.

5. Tonetti MS, Greenwell H, Kornman KS. Staging and grading of periodontitis: Framework and proposal of a new classification and case definition. J Periodontol. 2018;89:S159-S172.

6. Corbella S, Taschieri S, Del Fabbro M, Francetti L, Weinstein R, Ferrazzi E. Adverse pregnancy outcomes and periodontitis: A systematic review and meta-analysis exploring potential association. Quintessence Int. 2016;47:193-204.

7. Zini A, Sgan-Cohen HD, Marcenes W. Socio-economic position, smoking, and plaque: a pathway to severe chronic periodontitis. J Clin Periodontol. 2011;38:229-35.

8. König J, Holtfreter B, Kocher T. Periodontal health in Europe: future trends based on treatment needs and the provision of periodontal services--position paper 1. Eur J Dent Educ. 2010;14:4-24.

9. Burt B; Research, Science and Therapy Committee of the American Academy of Periodontology. Position paper: epidemiology of periodontal diseases. J Periodontol. 2005;76:1406-19.

10. Borrell LN, Crawford ND. Socioeconomic position indicators and periodontitis: examining the evidence. Periodontol 2000. 2012;58:6983.

11. Thomson WM, Sheiham A, Spencer AJ. Sociobehavioral aspects of periodontal disease. Periodontol 2000. 2012;60:54-63.

12. Joshi S, Suominen AL, Knuuttila M, Bernabé E. Toothbrushing behaviour and periodontal pocketing: An 11-year longitudinal study. J Clin Periodontol. 2018;45:196-203.

13. Marchesan JT, Morelli T, Moss K, Preisser JS, Zandona AF, Offenbacher S, Beck J. Interdental Cleaning Is Associated with Decreased Oral Disease Prevalence. J Dent Res. 2018;97:773-78.

14. Karimalakuzhiyil Alikutty F, Bernabé E. Long-term regular dental attendance and periodontal disease in the 1998 adult dental health survey. J Clin Periodontol. 2016;43:114-20.

15. Nociti FH Jr, Casati MZ, Duarte PM. Current perspective of the impact of smoking on the progression and treatment of periodontitis. Periodontol 2000. 2015;67:187-210.

16. Lertpimonchai A, Rattanasiri S, Arj-Ong Vallibhakara S, Attia J, Thakkinstian A. The association between oral hygiene and periodontitis: a systematic review and meta-analysis. Int Dent J. 2017;67:332-43. 17. World Health Organization. Oral Health Surveys: Basic Methods, 4th ed. Geneva: World Health Organization; 1997.

18. Mamai-Homata E, Polychronopoulou A, Topitsoglou V, Oulis C, Athanassouli T. Periodontal diseases in Greek adults between 1985 and 2005--risk indicators. Int Dent J. 2010;60:293-99.
19. Moller IJ, Marthaler T. National Oral Health Pathfinder Survey. Report on a visit to Greece. WHO regional office for Europe. Copenhagen: World Health Organization; 1988.

20. Mamai-Homata E, Topitsoglou V, Oulis C, Margaritis V, Polychronopoulou A. Risk indicators of coronal and root caries in Greek middle-aged adults and senior citizens. BMC Public Health. 2012;12:484. 21. Mamai-Homata E, Margaritis V, Koletsi-Kounari H, Oulis C, Polychronopoulou A, Topitsoglou V. Tooth loss and oral rehabilitation in Greek middle-aged adults and senior citizens. Int J Prosthodont. 2012;25:173-79.

22. Pourhoseingholi MA, Vahedi M, Rahimzadeh M. Sample size calculation in medical studies. Gastroenterol Hepatol Bed Bench. 2013;6:14-7.

23. Greene JC. The Oral Hygiene Index--development and uses. J Periodontol. 1967;38:625-37.

24. Bernabé E, Knuuttila M, Suominen AL. Interdental cleaning and periodontal pocketing among finnish adults. J Clin Periodontol. 2019;4:310-20.

\section{Consent to participate}

Informed consent was obtained from all involved subjects.

\section{Ethics}

Permission from the Greek Ministry of Health (\#143207/15-12-2012) was obtained before the clinical examinations.

Source of funding

The study was funded by a National Strategic Reference Framework (NSRF) Program (\#5402/29-05-2013).

\section{Authors' contributions}

ID contributed to the data analysis and interpretation, drafted the manuscript; AP contributed to the data analysis and interpretation, critically revised the manuscript; WP contributed to the study design, critically revised the manuscript; CT contributed to the data analysis and interpretation, critically revised the manuscript; VM contributed to the study design, critically revised the manuscript; EMH contributed to the study design, critically revised the manuscript; SK contributed to the study design, data analysis and interpretation and critically revised the manuscript.

Acknowledgments: The authors are indebted to all board members and coordinators of the local dental societies who, along with the calibrated examiners contributed to this Pan-Hellenic pathfinder survey, which was conducted under the auspices of the Hellenic Dental Association and the scientific coordination of Prof. C.J Oulis, in collaboration with the Dental Schools of Athens and Thessaloniki.

\section{Conflicts of Interest}

The authors state that there are no conflicts of interest to disclose. 